www.jmscr.igmpublication.org

ISSN (e)-2347-176x ISSN (p) 2455-0450

crossrefDOI: https://dx.doi.org/10.18535/jmscr/v7i1.95

Journal Of Medical Science And Clinical Research

IGM Publication

An Official Publication of IGM Publication

\title{
The Effect of Neuromuscular Taping in Improving Upper Extremity Functions in Children with Cerebral Palsy
}

\author{
Authors \\ Sujoy Roy ${ }^{1}$, Jaya Dixit ${ }^{2}$ \\ ${ }^{1}$ Occupational Therapist, Sir Sunderlal Hospital, Banaras Hindu University, Varanasi, Uttar Pradesh, India \\ ${ }^{2}$ Occupational Therapist, Sir Sunderlal Hospital, Banaras Hindu University, Varanasi, Uttar Pradesh, India \\ Ph number: 8756467856, Email: jayadixitmot@yahoo.com
}

Corresponding Author

Sujoy Roy

Ph number: 9476276333, Email: sujoyroy008@gmail.com

\begin{abstract}
Background: The purpose of this study was to examine the effect of application of Kinesiotape over the shoulder muscles to improve functions of upper extremity in children with cerebral palsy.

Objective: To find out the effect of kinesiotaping over the shoulder muscular along with conventional occupational therapy, in improving upper limb functions of children with cerebral palsy.

Method: It was a Pre Test and Post Test experimental study 60 children with cerebral palsy who were fulfilling the inclusion criteria were selected by convenient sampling. A written informed consent and ethical permission was obtained. Quality of upper extremity skill test (QUEST) was used as instrument for measuring improvement in upper extremity functions. Therapy for both the groups was given for 1 hour per session. In the experimental group along with conventional occupational therapy, kinesiotape (KT) was applied over the shoulder muscles.
\end{abstract}

Results: After intervention there was improvement in mean \& standard deviation of QUEST more in experimental group as compared to control group.

Conclusion: It can be concluded that application of kinesiotape along with conventional occupational therapy can be used to enhance \& improve upper extremity functions in children with cerebral palsy.

Keywords: Kinesiotape, Shoulder, QUEST, Cerebral Palsy.

\section{Introduction}

Cerebral palsy $(\mathrm{CP})$ is the most common cause of physical disability in childhood. It is a neurodevelopment disorder caused by nonprogressive lesion(s) in the immature brain implying spasticity, muscle weakness, decreased selective motor control and secondary musculoskeletal problems usually accompanied also by sensory and cognitive impairments (Bax $\mathrm{M}$ et al
2005). The upper limb is usually more severely involved than lower one in most types of cerebral palsy. (Tong-Wai $\mathrm{R}$ et al.2006). This limits reaching, grasping and object manipulation, interfering also with exploration, play, self-care and other activities of daily living (Jaspers E et al 2009).KT method which was first described by Dr. Kenzo Kasein 1996 is used to increase sensory stimulation, strengthen the weak muscles, 
inhibit spastic muscles, increase joint stability, increase functional motor skills, help with postural control and improve functional independence in pediatric rehabilitation clinics in addition to the occupational therapy programs (Kase K et al 2006 ) .This innovative taping application is based on eccentric stimulation of the skin, muscle tissue, tendons, neurological vessels, lymphatic and vascular pathways improving their functioning. Neuromuscular taping (NMT) provides passive stretching through the application of a tape with eccentrical properties encouraging flexibility and coordination and bettering range of movement in patients suffering with excessive muscle contraction due to different clinical conditions. It has been claimed that the effects may be due to the sensorimotor and proprioceptive feedback mechanisms. It has been hypothesized that the application of NMT is able to stimulate cutaneous mechanoceptors. These receptors activate nerve impulses when mechanical loads (touch, pressure, vibration, stretch and itch) create deformation. Their activation by an adequate stimulus causes local depolarization, which triggers nerve impulse along the afferent fibers travelling towards the central nervous system. However, few applications support the use of this type of tape to improve the upper-body functionality in $\mathrm{CP}$. KT consists of noninvasive adhesive elastic taping, the purpose of which is to mechanically restrain pathological movements, to preserve functional movements at the same time, and to enhance perceptive feedbacks (Iosa $M$ et al 2010\& Thornton J Let al 1996). The aim of this study is to use kinesiotape in facilitating movements of upper extremity in children with cerebral palsy.

\section{Methodology}

This study adhered to the tenets of Declaration of Helsinki guidelines. 60 children with medical diagnosis of cerebral palsy were enrolled in this study. The Ethical Committee of university approved our protocol .Children excluded from the study were those on Botox therapy (within the past 6 months) or muscle relaxants, those with active seizure disorders, those who had undergone any surgery for the upper limb, and those having allergic reaction to Kinesiotape. In all the subjects, shoulder muscles spasticity was lower than 2 on the Modified Ashworth Scale and passive shoulder range of motion was full, with preserved ability to actively move the shoulder in all directions. The study purpose and test procedure were elaborately explained to the parents/ guardians, after which written informed consent was obtained for their child's participation. A patch test with the Kinesio tape was performed on the dorsum of the hand (to confirm or rule out subject's allergy to Kinesio tape).

\section{Application of Kinesio tape}

Tape application was performed in a quiet environment with each child's being positioned comfortably on the chair. The skin was cleaned by Surgical Spirit 70\% alcohol, and then Kinesio tape was applied over the muscles belly of pectorialis major and deltoid i.e. from the upper chest to upper arm and upper back to upper arm using the "fanning" technique as shown in the figure 1 . The Kinesio tape was kept on the child's shoulder for 3 days. After 3 days KT was removed and area of application was left open for 24 hours. Then again it is applied for other 3 days. This sequence was carried out for a month.

\section{Results}

Pretest and Posttest scores were collected using all the components of QUEST and were added and summed up to find the raw score and final scores.

Table 1 shows that $60 \%$ of children with cerebral palsy who participated in the study were male which were 35 in number between the age range of 42-72 months and mean age of $57 \pm 1.23$ months. There were 25 females which are $40 \%$ of total population in the study with the age range of 36-69 months and mean age and standard deviation of 52.5 \pm 2.80 .During the study we encountered 4 types of cerebral palsy children with 43 spastic diplegic, 6 spastic hemiplegic, 4 spastic quadriplegic and 7 athetoid. Table 2 shows the mean and standard deviation of subtest 
dissociated movement of QUEST which shows that there was improvement in mean and standard deviation of shoulder, elbow, wrist, finger and grasp in both experimental and control group with more improvement in experimental group. Table 3 shows that there is much improvement in position of body component of grasp subtest, but there is also improvement in other 2 components of grasp 1 cube and cereal. Table 4 shows the improvement in mean and standard deviation of weight bearing in quadruped and weight bearing with reaching in this position. Table 5 shows that there is more improvement in mean and standard deviation of experimental group for protective extensions, hand function, spasticity and child seems to be more cooperative for therapy in experimental group as compared to control group according to the mean and standard deviation .Table 6 shows the results of wilcoxan sign rank test for subtest A dissociation of movement in QUEST were $\mathrm{Z}$ and $\mathrm{P}$ for experimental group is -2.877 and 0.004 respectively with level of confidence interval value of $95 \% .5 .34$ as lower limit and 10.67 as upper limit .Control group is having $\mathrm{Z}$ value of 2.44 and $P$ value 0.014 with lower limit 4.16 and upper limit 7.89 and level of confidence interval value of $95 \%$. Table 7 shows results of wilcoxan signed rank test for subtest $B$ i.e. grasp were experimental group is having $\mathrm{Z}$ value of -1.17 and $P$ value of 0.004 with $95 \%$ confidence inertial value ranging from 1.34 to $1.67 . \mathrm{Z}$ and $\mathrm{P}$ of -1.44 and 0.014 respectively for control group with 95\% confidence interval value and lower limit of 1.16 and upper limit of 1.89 .Table 8 wilcoxan signed rank test for component $\mathrm{C}$ weight bearing shows improvement in both the group the control and experimental group with $\mathrm{Z}$ value of -2.12 and -2.29 respectively for experimental and control group and $\mathrm{p}$ value of 0.004 and 0.014 respectively for experimental and control group .

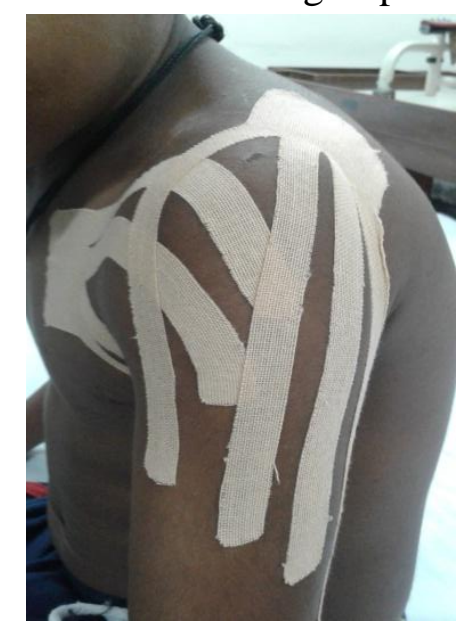

Figure 1 showing pattern of kinesiotaping over the shoulder joint

Table 1. Demographic details of children included in the study.

\begin{tabular}{|l|c|c|}
\hline Gender & Male & $60 \%(35)$ \\
& female & $40 \%(25)$ \\
\hline Age range & Male & $42-72$ months \\
& female & $36-69$ months \\
\hline Dominance & Right & $40 \% ; \mathrm{n}=24$ \\
& Left & $60 \% ; \mathrm{n}=36$ \\
\hline Involved hand & Right & $46.2 \% ; \mathrm{n}=28$ \\
& Left & $53.8 \% ; \mathrm{n}=32$ \\
\hline Type of cerebral palsy & Spastic diplegic & 43 \\
& Spastic hemiplegic & 6 \\
& Quadriplegic & 4 \\
& Athetoid & 7 \\
\hline
\end{tabular}


Table 2.Mean and standard deviation of Dissociated movement subtest of QUEST.

\begin{tabular}{|l|c|c|}
\hline Component A & Mean \pm std in control group & Mean \pm std in experimental group \\
\hline Shoulder & $38.03 \pm 9.61$ & $44.19 \pm 6.80$ \\
\hline Elbow & $5.40 \pm 10.17$ & $20.2 \pm 22.98$ \\
\hline Wrist & $22.03 \pm 14.67$ & $36.73 \pm 16.04$ \\
\hline Finger & $88.50 \pm 24.04$ & $99.03 \pm 21.74$ \\
\hline Grasp & $98.42 \pm 22.86$ & $98.95 \pm 13.12$ \\
\hline
\end{tabular}

Table 3 Mean and standard deviation of grasp subtest of QUEST.

\begin{tabular}{|l|c|c|}
\hline Component B & Mean \pm std in control group & Mean \pm std in experimental group \\
\hline Position of body & $88.50 \pm 24.04$ & $99.03 \pm 21.74$ \\
\hline Grasp of 1" cube & $96.42 \pm 22.86$ & $97.95 \pm 13.12$ \\
\hline Grasp of cereal & $91.03 \pm 21.74$ & $92.92 \pm 10.90$ \\
\hline
\end{tabular}

Table 4 Mean and standard deviation of weight bearing subtest of QUEST

\begin{tabular}{|l|c|c|}
\hline Component C & Mean \pm std in control group & Mean \pm std in experimental group \\
\hline Weight bearing & $23.80 \pm 10.04$ & $28.80 \pm 11.03$ \\
\hline Weight bearing with reaching & $60.92 \pm 11.49$ & $71.84 \pm 16.52$ \\
\hline
\end{tabular}

Table 5 Mean and standard deviation of subtest D, E, F, G of QUEST

\begin{tabular}{|l|c|c|}
\hline Components & Mean \pm std in control group & Mean \pm std in experimental group \\
\hline D. Protective extension & $60.20 \pm 11.42$ & $72.19 \pm 16.2$ \\
\hline E. Hand function & $71.84 \pm 16.52$ & $72.85 \pm 17.41$ \\
\hline F. spasticity & $55.19 \pm 16.22$ & $59.04 \pm 9.60$ \\
\hline G. Cooperativeness & $51.90 \pm 9.12$ & $49.90 \pm 6.12$ \\
\hline
\end{tabular}

Table 6 Results of Wilcoxon Signed Rank Test for subtest A dissociation of movement in QUEST

\begin{tabular}{|l|c|c|c|c|}
\hline Groups & $\boldsymbol{Z}(\mathbf{2}$ tailed $)$ & $\boldsymbol{P}(\mathbf{2}$ tailed $)$ & \multicolumn{2}{|c|}{$\mathbf{9 5 \%}$ Confidence Interval Value } \\
\cline { 4 - 5 } & & & Lower Limit & Upper Limit \\
\hline Experimental & -2.877 & 0.004 & 5.34 & 10.67 \\
\hline Control & -2.449 & 0.014 & 4.16 & 7.89 \\
\hline
\end{tabular}

Table 7 Results of Wilcoxon Signed Rank Test for component B grasp subtest of QUEST

\begin{tabular}{|l|c|c|c|c|}
\hline Groups & $\boldsymbol{Z}(\mathbf{2}$ tailed $)$ & $\boldsymbol{P}(\mathbf{2}$ tailed $)$ & \multicolumn{2}{|c|}{$\mathbf{9 5 \%}$ Confidence Interval Value } \\
\cline { 3 - 5 } & & & Lower Limit & Upper Limit \\
\hline Experimental & -1.17 & 0.004 & 1.34 & 1.67 \\
\hline Control & -1.44 & 0.014 & 1.16 & 1.89 \\
\hline
\end{tabular}

Table 8 Results of Wilcoxon Signed Rank Test for component C weight bearing subtest of QUEST

\begin{tabular}{|l|c|c|c|c|}
\hline Groups & $\boldsymbol{Z}(\mathbf{2}$ tailed $)$ & $\boldsymbol{P}(\mathbf{2}$ tailed $)$ & \multicolumn{2}{|c|}{$\mathbf{9 5 \%}$ Confidence Interval Value } \\
\cline { 4 - 5 } & & & Lower Limit & Upper Limit \\
\hline Experimental & -2.12 & 0.004 & 4.43 & 9.07 \\
\hline Control & -2.29 & 0.014 & 4.61 & 7.19 \\
\hline
\end{tabular}

Table 9 Results of Wilcoxon Signed Rank Test of subtest D, E, F, G of QUEST

\begin{tabular}{|l|c|c|c|c|}
\hline Groups & $\boldsymbol{Z}(\mathbf{2}$ tailed $)$ & $\boldsymbol{P}(\mathbf{2}$ tailed $)$ & \multicolumn{2}{|c|}{$\mathbf{9 5 \%}$ Confidence Interval Value } \\
\cline { 4 - 5 } & & & Lower Limit & Upper Limit \\
\hline Experimental & -1.06 & 0.004 & 2.34 & 8.67 \\
\hline Control & -1.14 & 0.014 & 2.16 & 5.89 \\
\hline
\end{tabular}




\section{Discussion}

This paper investigates the effect of kinesiotape over the muscle belly of deltoid and pectoralis major muscles in functional outcome of upper limbs of children with cerebral palsy. The results suggest that the Kinesio tape may improve upper motor function in children with CP. Kinesiotaping enhances the kinesthetic inputs and facilitates enhanced control in the shoulder muscles to improve volitional control of the muscle and tendon movement during activities, thereby improving the functional control and movement of upper limbs, so improving fine motor hand function (Yasukawa A et al 2006). We assume that extension of the tape from chest to shoulder and from upper back to upper arm might have enhanced shoulder stability, which probably would have improved shoulder and upper arm muscle activity, hence facilitating better shoulder movement and function (Kase K et al 2003). Because the tape was retained for a month over the involved shoulder of children with $\mathrm{CP}$, we assume that the anticipatory control due to the presence of the tape over the shoulder and arm muscles would have induced better muscular coordination and hence improved upper limb motor skills and hand functions. Because the kinesiotape was applied over the shoulder muscles starting from their origin and extended up to the upper area of arm till the insertion of muscles, covering the shoulder and arms, we believe that such techniques would have provided improved stability. This would have facilitated clinically positive changes in the upper limb motor functions and functions of hands. This comes in consistent with Murray 2005, who reported that neuromuscular tapping as an adjunct to the therapeutic procedures can improve strength, functional activities, proprioception, control and positioning. KT increases blood circulation in the taped area (Ogura1998; Oliveria 1999; Vorhies 1999; Wallis 1999; Kase 1994; Kase and Hashimoto 2005; Murray 2005), and this physiological change may affect the muscle and myofascia functions after the application of neuromuscular tapping helping the children to generate the necessary force required for the function. An additional theory is that neuromuscular tapping stimulates sensory receptors and cutaneous mechanoreceptors at the taped area. Cutaneous mechanoreceptors activate nerve impulses when mechanical loads create deformation. The activation of cutaneous mechanoreceptors by an adequate stimulus causes local depolarizations that trigger nerve impulses along the afferent fiber traveling toward the central nervous system (Garcia 2001; Goo 2001; Halseth et al. 2004; Maruko 1999; Mori 2001; Murray and Husk 2001; Ogura1998; Vorhies 1999; Wallis 1999; Kase et al. 2003). The application of KT may apply pressure to the skin or stretch the skin, and this external load may stimulate cutaneous mechanoreceptors causing physiological changes in the taped area. Studies previously conducted to determine the effects of neuromuscular tapping on cutaneous mechanoreceptors (Garcia 2001; Goo 2001; Halseth et al. 2004; Maruko 1999; Mori 2001; Murray and Husk 2001; Ogura 1998; Vorhies 1999; Wallis 1999) have reported that neuromuscular tapping on select muscles and joints may improved muscle excitability. There is no study in the literature investigating the use of neuromuscular tape application over the shoulder and arm in improving upper limb motor functions among children with cerebral palsy.

\section{Conclusion}

It can be concluded that application of kinesiotape along with conventional occupational therapy can enhance and improve functions of upper extremity among children with cerebral palsy, so that they can have the functional independence, to meet demands of everyday life.

\section{Acknowledgement}

I thank my patients and their parents for trusting me, and their cooperation during the course of study. Above all I thank God Almighty for providing me all that I wanted and much more to carry out my study. 


\section{References}

1. Bax M Goldstein M, Rosenbaum P et al (2005). Executive Committee for the Definition of Cerebral Palsy. Proposed definition and classification of cerebral palsy. Dev Med Child Neurol; 47: 571 576.

2. Garcia D (2001) Kinesio taping for the sense of balance on knee [in Japanese]. $16^{\text {th }}$ Annual Kinesio Taping International Symposium Review, 20-23.

3. Goo J (2001) A new step for treatment of ankle sprain [in Japanese]. 16th Annual Kinesio Taping International Symposium Review, 16-19.

4. Halseth T, McChesney JW, DeBeliso M, Vaughn R, Lien, J (2004) The effects of kinesio taping on proprioception at the ankle. Journal of Sports Science and Medicine 3: 1-7.

5. Iosa M , Morelli D, Nanni M V et al. Functional taping: a promising technique for children with cerebral palsy. Dev Med Child Neurol 2010; 52: 587 - 589.

6. Kase K (1994) Illustrated Kinesio-Taping. 2nd ed. Tokyo, Japan: Ken'i-kai Information. 6-9.

7. Kase K, Hashimoto T, Okane T (1996) Kinesio taping perfect manual: Amazing taping therapy to eliminate pain and muscle disorders. Albuquerque, NM: KMS, LLC.

8. Kase K ,Martin P, Yasukawa A (2006). Kinesiotaping in pediatrics.Fundamentals and whole body taping. Kinesio Taping Association, Albuquerque, New Mexico, USA; 9-30.

9. Murray H, Husk L (2001) Effect of kinesio taping on proprioception in the ankle.Journal of Orthopedic Sports Physical Therapy 31: A-37.

10. Maruko K (1999) Aqua therapy using kinesio taping of central cooperation disabled pediatrics [in Japanese]. 15th Annual Kinesio Taping International Symposium Review. 47-54, 70-73.

11. Mori S (2001) How Kinesio taping method can induce effectiveness for treatment of scapular arch [in Japanese]. 16th Annual Kinesio Taping International Symposium Review. 50-53.

12. Murray H, Husk L (2001) Effect of kinesio taping on proprioception in the ankle. Journal of Orthopedic Sports Physical Therapy 31: A-37.

13. Ogura R (1998) Overuse syndrome for long-distance runners and taping [in Japanese]. 14th Annual Kinesio Taping International Symposium Review. 51-52.

14. Oliveria R (1999) Soft tissue injuries in sports people-The contribution of kinesiotaping [in Japanese]. 15th Annual Kinesio Taping International Symposium Review.13-23.

15. Tong-Wai R, Webster $\mathrm{R}$ and Shevel $\mathrm{M}$ (2006): A clinical and etiologic profile of spastic diplegia.Pediatric Neurology. 34(3): 212-218.

16. Vorhies, D (1999) Testimonial of a kinesiotape convert [in Japanese]. 15th Annual Kinesio Taping International Symposium Review. 122-123.

17. Wallis J (1999) Effects of kinesio taping on pain perception of athletes with patellofe pain syndrome A pilot study [in Japanese]. 15th Annual Kinesio Taping International Symposium Review. 44-46.

18. Yasukawa A et al, Patel P, Sisung C (2006). Pilot study: investigating the effects of kinesio taping in an acute pediatric rehabilitation setting. Am J Occup Ther.; 60:104-10. 